\title{
An NSFD scheme for SIR epidemic models of childhood diseases with constant vaccination strategy
}

\author{
Qianqian Cui ${ }^{1,2}$, Jiabo Xu ${ }^{3}$, Qiang Zhang ${ }^{2}$ and Kai Wang ${ }^{4 *}$
}

*Correspondence:

wangkaimath@sina.com

${ }^{4}$ Department of Medical

Engineering and Technology, Xinjiang Medical University, Urumqi,

830011, People's Republic of China

Full list of author information is

available at the end of the article

\begin{abstract}
In this paper, we construct a nonstandard finite difference (NSFD) scheme for an SIR epidemic model of childhood disease with constant strategy. The dynamics of the obtained discrete model is investigated. First we show that the discrete model has equilibria which are exactly the same as those of the continuous model. Furthermore, we prove that the conditions for those equilibria to be globally asymptotically stable are consistent with the continuous model for any size of numerical time-step. The analytical results are confirmed by some numerical simulations.
\end{abstract}

Keywords: mathematical model; transmission dynamics; basic reproduction number; sensitivity analysis; control strategies

\section{Introduction}

Childhood diseases are the most common form of infectious diseases. These are the diseases such as measles, mumps, chicken pox, rubella, poliomyelitis, etc. to which children are born susceptible and usually contract within five years. Because young children are in frequent contact with each other at school or other place, such a disease can be spread very quickly. Meanwhile, the development of vaccines against infectious children diseases has been booming and protecting children from the diseases. Hence vaccination is considered to be the most effective strategy against childhood diseases, it is essential for us to predict the optimal vaccine coverage level to prevent the spread of theses diseases. A universal effort to extend vaccination coverage to all children began in 1974, when the World Health Organization (WHO) founded the Expanded Program on Immunization (EPI). Mathematical models (see [1-7]) of deterministic type have often been used to provide deeper insights into the transmission dynamics of a childhood disease and to evaluate control strategies.

In this paper, the total population that is involved in the spread of infection is split into three epidemiological classes: a susceptible class $(S)$, an infected class $(I)$ and a removed class $(R)$ denoting vaccinated as well as recovered people with permanent immunity. We assume that the efficacy of vaccine is $100 \%$, and the natural death rates $\mu$ in the classes remain unequal to births, so that the total population $N$ is realistically not constant. Citizens are born into the population at a constant birth rate $A$ with extremely low childhood disease mortality rate. We denote the fraction of citizens vaccinated at birth each year as $p$ 
(with $0<p<1$ ) and assume the rest are susceptible. A susceptible individual will move into the infected group through contact with an infected individual, approximated by an average contact rate $\beta$. An infected individual recovers at rate $\gamma$, and enters the removed class. The removed class also contains people who are vaccinated. The differential equations for the SIR (see $[1,4,7])$ epidemic model of childhood diseases with constant vaccination strategy are as follows:

$$
\left\{\begin{array}{l}
\frac{d S}{d t}=(1-p) A-\beta S I-\mu S, \\
\frac{d I}{d t}=\beta S I-(\mu+\gamma) I, \\
\frac{d R}{d t}=p A+\gamma I-\mu R .
\end{array}\right.
$$

The biological background requires that all parameters be nonnegative. Makinde [4] employed the Adomian decomposition method to compute an approximate non-perturbative solutions of model (1.1). Yildirim and Cherruault [7] by qualitative analysis revealed the vaccination reproductive number for disease control and eradication.

However, for practical purposes, it is often necessary to discretize the continuous model. The discrete dynamical system obtained from the discretization should contain as many qualitative properties of the continuous problem as possible. It is shown that many standard methods such as Euler method, Runge-Kutta method and some other standard finite schemes implemented in a dynamical system can lead to negative solutions for spurious dynamical behaviors such as converging to wrong equilibrium point or wrong periodic cycle or numerical instabilities [8-10]. In this paper, we propose a numerical scheme to solve model (1.1) by implementing a nonstandard finite difference (NSFD) scheme. This method was originally developed by Mickens [11-16]. The nonstandard scheme relied on the following important rules: the standard denominator $h$ in standard discrete derivative is replaced by a denominator function $0<\phi(h)<1$, where $\phi(h)=h+o\left(h^{2}\right)$; the nonlinear terms are approximated in a nonlocal way using more than one mesh point. Here, $h$ is the timestep size of numerical integration. Moreover, the fundamental principle for constructing NSFD scheme for differential equations is dynamic consistency, that is, the discretized model maintain essential dynamical properties such as positivity of solutions, boundedness of solutions, monotonicity of solutions, correct number and stability of fixed-points and other special solutions of the continuous model. This method has been applied to various problems in which the resulting discrete systems preserve dynamical properties of the related continuous models [5, 17-19]. In [19], the NSFD scheme has been implemented in a special class of SIR epidemic models. Mickens [5] considered a SIR epidemic model with square-root dynamics.

This paper is organized as follows. In the next section, we present several important properties of solutions to the continuous model. A particular discretization is constructed in Section 3. We illustrate the global asymptotic stability of disease-free equilibrium and endemic equilibrium in Sections 4 and 5, and provided numerical examples to verify our results in Section 6. Finally, we provide a summary of the obtained results and present a possible extension of this work.

\section{Dynamical properties of the continuous model}

In this section, we review some dynamical properties of the SIR epidemic model with constant vaccination strategy (1.1). It should be noted that the system of continuous equations, 
if added together, satisfies the conservation law [16]

$$
\frac{d N}{d t}=A-\mu N, \quad N(t)=S(t)+I(t)+R(t) .
$$

The exact solution of equation (2.1) is

$$
N(t)=\frac{A}{\mu}+\left(N(0)-\frac{A}{\mu}\right) e^{-\mu t},
$$

where $N(0)=S(0)+I(0)+R(0)$. Any solution $(S(t), I(t), R(t)), t \geq 0$, of model (1.1) satisfies

$$
\begin{aligned}
& S(0) \geq 0, \quad I(0) \geq 0, \quad R(0) \geq 0 \\
& \Rightarrow \quad S(t) \geq 0, \quad I(t) \geq 0, \quad R(t) \geq 0 .
\end{aligned}
$$

All valid epidemic models must have this feature since negative population numbers cannot exist as a physical reality. Moreover, it is easy to verify that the domain

$$
D=\left\{(S, I, R) \in R_{+}^{3} \mid S+I+R \leq A / \mu\right\}
$$

is a compact, positively invariant set for model (1.1).

Define the basic reproduction number as follows:

$$
\mathcal{R}_{0}=\frac{(1-p) \beta A}{\mu(\mu+\gamma)}
$$

Then the following results can be summarized in Li et al. [3].

Theorem 1 Model (1.1) always has a disease-free equilibrium $E^{0}=((1-p) A / \mu, 0, p A / \mu)$ and has a unique endemic equilibrium $E^{*}=\left(S^{*}, I^{*}, R^{*}\right)$ when $\mathcal{R}_{0}>1$, where

$$
S^{*}=\frac{\mu+\gamma}{\beta}, \quad I^{*}=\frac{\mu\left(\mathcal{R}_{0}-1\right)}{\beta}, \quad R^{*}=\frac{p A+\gamma I}{\mu} .
$$

Theorem 2 For model (1.1), the following results hold.

(i) If $\mathcal{R}_{0}<1$, the disease-free equilibrium $E^{0}$ is globally asymptotically stable in D. On the other hand, if $\mathcal{R}_{0}>1, E^{0}$ is unstable;

(ii) If $\mathcal{R}_{0}>1$, then the unique endemic equilibrium $E^{*}$ is globally asymptotically stable in $D$.

\section{The NSFD scheme}

To derive the NSFD scheme, we define the following notation [16]:

$$
t \rightarrow t_{n}=n h, \quad(S(t), I(t), R(t)) \rightarrow\left(S_{n}, I_{n}, R_{n}\right), \quad n=0,1,2, \ldots,
$$

where $h>0$ is the constant time-step size. By applying the NSFD scheme to model (1.1), we can obtain the following discrete-time SIR epidemic model with constant vaccination 
strategy

$$
\left\{\begin{array}{l}
\frac{S_{n+1}-S_{n}}{\phi}=(1-p) A-\beta S_{n+1} I_{n}-\mu S_{n+1} \\
\frac{I_{n+1}-I_{n}}{\phi}=\beta S_{n+1} I_{n}-(\gamma+\mu) I_{n+1} \\
\frac{R_{n+1}-R_{n}}{\phi}=p A+\gamma I_{n+1}-\mu R_{n+1}
\end{array}\right.
$$

where the denominator function $\phi=\phi(h, \mu)($ see $[11,12])$ is

$$
\phi(h, \mu)=\frac{\mathrm{e}^{\mu h}-1}{\mu} .
$$

It is noted that discretized model (3.1) can be considered as using the standard Euler method for the first derivative and a nonlocal expression for nonlinear terms. It can be easily rearranged to get its explicit version

$$
\left\{\begin{array}{l}
S_{n+1}=\frac{S_{n}+(1-p) \phi A}{1+\phi\left(\beta I_{n}+\mu\right)} \\
I_{n+1}=\frac{I_{n}+\beta \phi S_{n+1} I_{n}}{1+(\gamma+\mu) \phi} \\
R_{n+1}=\frac{R_{n}+p \phi A+\gamma \phi I_{n+1}}{1+\mu \phi} .
\end{array}\right.
$$

Since all the parameters in model (3.2) are positive, it is clear that if the initial values $S_{0}, I_{0}$ and $R_{0}$ are positive, then the numerical solutions will also be positive for all $n \geq 0$, namely

$$
\begin{aligned}
S_{0} \geq 0, \quad I_{0} \geq 0, \quad R_{0} \geq 0 \\
\Rightarrow \quad S_{n} \geq 0, \quad I_{n} \geq 0, \quad R_{n} \geq 0, \quad n=0,1,2, \ldots .
\end{aligned}
$$

Defining $N_{n}=S_{n}+I_{n}+R_{n}$ and adding the three equations of model (3.1), we get

$$
\frac{N_{n+1}-N_{n}}{\phi}=A-\mu N_{n+1}, \quad N_{0}=S_{0}+I_{0}+R_{0},
$$

which is the exact finite scheme for the conservation law as expressed by equation (2.1) (see [16]). A straightforward calculation gives

$$
N_{n}=N\left(t_{n}\right)=\frac{1}{\mu}\left(A-\left(A-\mu N_{0}\right) \exp (-\mu n h)\right) .
$$

Similar to the continuous-time case, discrete model (3.1) or equivalent (3.2) also has a compact, positivity invariant set

$$
D_{h}=\left\{\left(S_{n}, I_{n}, R_{n}\right) \in \mathrm{R}_{+}^{3} \mid S_{n}+I_{n}+R_{n} \leq A / \mu, n=0,1,2, \ldots\right\} .
$$

It is easy to verify that discrete model (3.1) or equivalent (3.2) has the same equilibrium as model (1.1) which is independent of $h$. It can be described as the following theorem.

Theorem 3 For model (3.1) or equivalent (3.2), there always exists a disease-free equilibrium $E_{d}^{0}=\left(S_{d}^{0}, I_{d}^{0}, R_{d}^{0}\right)=((1-p) A / \mu, 0, p A / \mu)$ and has a unique endemic equilibrium $E_{d}^{*}=\left(S_{d}^{*}, I_{d}^{*}, R_{d}^{*}\right)$ when $\mathcal{R}_{0}>1$, where

$$
S_{d}^{*}=\frac{\mu+\gamma}{\beta}, \quad I_{d}^{*}=\frac{\mu\left(\mathcal{R}_{0}-1\right)}{\beta}, \quad R_{d}^{*}=\frac{p A+\gamma I}{\mu} .
$$




\section{Global asymptotic stability of disease-free equilibrium $E_{d}^{0}$}

In this section, we mainly discuss the global asymptotic stability of disease-free equilibrium $E_{d}^{0}$. First, we consider the local stability of $E_{d}^{0}$ for model (3.1). In order to obtain the local stability of equilibria for discrete model (3.1) or equivalent (3.2), we notice that the first two equations in model (3.1) or (3.2) do not depend on the third equation, and therefore the third equation can be omitted without loss of generality properties. For the sake of simplicity, we define the following functions [19]:

$$
\begin{aligned}
& F(S, I)=\frac{S+(1-p) \phi A}{1+\phi(\beta I+\mu)}, \\
& G(S, I)=\frac{I+\beta \phi F(S, I)}{1+(\mu+\gamma) \phi} .
\end{aligned}
$$

Obviously, the Jacobian matrix $[20,21]$ at the equilibrium point $(\tilde{S}, \tilde{I})$ is given by

$$
J(\tilde{S}, \tilde{I})=\left(\begin{array}{ll}
\frac{\partial F}{\partial S}(\tilde{S}, \tilde{I}) & \frac{\partial F}{\partial I}(\tilde{S}, \tilde{I}) \\
\frac{\partial G}{\partial S}(\tilde{S}, \tilde{I}) & \frac{\partial G}{\partial I}(\tilde{S}, \tilde{I})
\end{array}\right) .
$$

Now, we give the following theorem about the local stability of the disease-free equilibrium $E_{d}^{0}$ for model (3.1).

Theorem 4 If $\mathcal{R}_{0}<1$, the disease-free equilibrium point $E_{d}^{0}$ of discrete model (3.1) or equivalent (3.2) is locally asymptotically stable in $D_{h}$. On the other hand, if $\mathcal{R}_{0}>1, E_{d}^{0}$ is unstable.

Proof Substituting the disease-free equilibrium $E_{d}^{0}$ to Jacobian matrix (4.1) yields

$$
J\left(S_{d}^{0}, I_{d}^{0}\right)=\left(\begin{array}{cc}
\frac{1}{1+\mu \phi} & -\frac{1-p A}{\mu(1+\mu \phi)} \\
0 & \frac{1+\beta \phi(1-p) A / \mu}{1+(\mu+\gamma) \phi}
\end{array}\right) .
$$

The eigenvalues of $J\left(S_{d}^{0}, I_{d}^{0}\right)$ are as follows:

$$
\lambda_{1}=\frac{1}{1+\mu \phi}, \quad \lambda_{2}=\frac{1+\beta \phi(1-p) A / \mu}{1+(\mu+\gamma) \phi} .
$$

Obviously $\left|\lambda_{1}\right|<1$ for all $h$. From the definition of basic reproductive number (2.4), we can easily conclude that $\mathcal{R}_{0}<1$ is equivalent to $(1-p) \beta A<\mu(\mu+\gamma)$. Thus, if $\mathcal{R}_{0}<1$, then the magnitude of eigenvalue $\lambda_{2}$ is also strictly less than unity irrespective of $h$. This completes the proof.

Next, let us consider the global asymptotic stability of disease-free equilibrium $E_{d}^{0}$ for model (3.1). Since the variable $R$ does not appear in the first and the second equations, it is sufficient to consider the following 2-dimensional system:

$$
\left\{\begin{array}{l}
\frac{S_{n+1}-S_{n}}{\phi}=(1-p) A-\beta S_{n+1} I_{n}-\mu S_{n+1}, \\
\frac{I_{n+1}-I_{n}}{\phi}=\beta S_{n+1} I_{n}-(\gamma+\mu) I_{n+1} .
\end{array}\right.
$$

Set

$$
\underline{S}=\liminf _{n \rightarrow \infty} S_{n}, \quad \bar{S}=\limsup _{n \rightarrow \infty} S_{n}, \quad \underline{I}=\liminf _{n \rightarrow \infty} I_{n}, \quad \bar{I}=\limsup _{n \rightarrow \infty} I_{n} .
$$


Similar to the Izzo et al. [22, proof of Lemma 3.3], we obtain the following basic lemma.

Lemma 1 For any solution $\left(S_{n}, I_{n}\right)$ of model (4.2), with the initial conditions $S_{0}>0, I_{0}>0$, we have that

$$
\left\{\begin{array}{l}
0<\frac{(1-p) A}{\mu+\beta \bar{I}} \leq \underline{S} \leq \bar{S} \leq \frac{(1-p) A}{\mu+\beta \underline{I}} \leq \frac{(1-p) A}{\mu}, \\
\frac{\beta \bar{S}}{\gamma+\mu} \geq 1 \quad \text { if } \bar{I}>0 \\
\frac{\beta \underline{S}}{\gamma+\mu} \leq 1 \quad \text { if } \underline{I}>0 .
\end{array}\right.
$$

Furthermore, we define the function $P(s)$ as follows:

$$
P(s) \equiv \frac{\beta s}{\mu+\gamma},
$$

then we easily get the following lemma.

Lemma $2 P(s)$ is strictly monotone increasing function on $[0,+\infty)$ with

$$
P(0)=0, \quad \mathcal{R}_{0}=P\left(\frac{(1-p) A}{\mu}\right)
$$

and

$$
\begin{cases}P(\bar{S}) \geq 1 \quad \text { if } \bar{I}>0 \\ P(\underline{S}) \leq 1 \quad \text { if } \underline{I}>0\end{cases}
$$

Moreover, if $\mathcal{R}_{0}>1$, then there exists a unique solution $S=S_{d}^{*}$ of $P(s)=1$ such that

$$
0<S_{d}^{*}=\frac{\mu+\gamma}{\beta}<\frac{(1-p) A}{\mu} .
$$

By applying techniques in Izzo et al. [22], we now prove the global stability of the diseasefree equilibrium for $\mathcal{R}_{0} \leq 1$.

Theorem 5 If $\mathcal{R}_{0} \leq 1$, then the disease-free equilibrium $E_{d}^{0}$ of model (3.1) is globally asymptotically stable.

Proof From (4.3) in Lemma 1, for any $\epsilon>0$, there exists an integer $n_{0}$ such that

$$
S_{n+1} \leq \frac{(1-p) A}{\mu}+\epsilon \text { for } n \geq n_{0} .
$$

Construct the following sequence $\left\{V_{n}\right\}_{n=0}^{+\infty}$ defined by

$$
V_{n}=I_{n}+\phi \beta S_{n+1} I_{n}
$$

Then, for $n \geq n_{0}$, we have

$$
\begin{aligned}
V_{n+1}-V_{n} & =I_{n+1}-I_{n}+\phi \beta S_{n+2} I_{n+1}-\phi \beta S_{n+1} I_{n} \\
& =\phi \beta S_{n+1} I_{n}-\phi(\gamma+\mu) I_{n+1}-\phi \beta S_{n+1} I_{n}+\phi \beta S_{n+2} I_{n+1}
\end{aligned}
$$




$$
\begin{aligned}
& =\phi \beta S_{n+2} I_{n+1}-\phi(\gamma+\mu) I_{n+1} \\
& \leq \phi \beta\left(\frac{(1-p) A}{\mu}+\epsilon\right) I_{n+1}-\phi(\gamma+\mu) I_{n+1} \\
& =\phi\left(\frac{\beta A(1-p)}{\mu}-(\gamma+\mu)+\beta \epsilon\right) I_{n+1} \\
& =\frac{\phi}{\gamma+\mu}\left(\mathcal{R}_{0}-1+\frac{\beta \epsilon}{\gamma+\mu}\right) I_{n+1} .
\end{aligned}
$$

Since $\epsilon$ is arbitrary, we conclude that if $\mathcal{R}_{0} \leq 1$, then

$$
V_{n+1}-V_{n} \leq \frac{\phi}{\gamma+\mu}\left(\mathcal{R}_{0}-1\right) I_{n+1} \leq 0,
$$

which yields that the sequence $\left\{V_{n}\right\}_{n=n_{0}}^{+\infty}$ is monotone decreasing. Therefore, there exists a nonnegative constant $\hat{V}$ such that $\lim _{n \rightarrow+\infty}=\hat{V}$. We will prove that $\hat{V}=0$ for $\mathcal{R}_{0} \leq 1$. In fact, if $\mathcal{R}_{0}<1$, then we have $\lim _{n \rightarrow+\infty} I_{n}=0$. Consequently, from (4.5), we have that $\hat{V}=0$. Meanwhile, by Lemma 1 , we can conclude that $\lim _{n \rightarrow+\infty} S_{n}=\frac{(1-p) A}{\mu}$. Suppose that $\mathcal{R}_{0}=1$, that is, $(1-p) A \beta=\mu(\mu+\gamma)$. We first transform model (4.2) into the following form:

$$
\left\{\begin{array}{l}
S_{n+1}=\frac{(1-p) A}{1+\phi \mu}+\frac{S_{n}}{1+\phi \mu}-\frac{\phi \beta S_{n+1} I_{n}}{1+\phi \mu} \\
I_{n+1}=\frac{I_{n}}{1+\phi(\mu+\gamma)}+\frac{\phi \beta S_{n+1} I_{n}}{1+\phi(\mu+\gamma)}
\end{array}\right.
$$

We claim that there exists a sequence $\left\{n_{k}\right\}$ such that $\lim _{k \rightarrow \infty} I_{n_{k}}=0$ if $\bar{I}=0$, the claim is evident. Now we consider the case $\bar{I}>0$. By applying the first inequality in (4.4), we obtain $\bar{S}=\frac{(1-p) A}{\mu}$. Therefore, there exists a sequence $\left\{n_{k}\right\}$ such that $\lim _{k \rightarrow+\infty} S_{n_{k}+1}=\bar{S}$. From the first equation of (4.6), we have

$$
S_{n_{k}+1}=\frac{(1-p) A}{1+\phi \mu}+\frac{S_{n_{k}}}{1+\phi \mu}-\frac{\phi \beta S_{n_{k}+1} I_{n_{k}}}{1+\phi \mu},
$$

that is,

$$
S_{n_{k}+1}=\bar{S}-\frac{S_{n_{k}+1}-S_{n_{k}}+\phi \beta S_{n_{k}+1} I_{n_{k}}}{\mu \phi} .
$$

As $k \rightarrow+\infty$, we obtain

$$
\lim _{k \rightarrow+\infty}\left(S_{n_{k}+1}-S_{n_{k}}+\phi \beta S_{n_{k}+1} I_{n_{k}}\right)=0,
$$

from which it is not difficult to obtain

$$
\lim _{k \rightarrow+\infty} S_{n_{k}+1}=\lim _{k \rightarrow+\infty} S_{n_{k}}=\bar{S}
$$

and

$$
\lim _{k \rightarrow+\infty} I_{n_{k}}=0
$$


We easily find that (4.7) implies $\lim _{n \rightarrow+\infty} S_{n}=\bar{S}=\frac{(1-p) A}{\mu}$. Meanwhile, combining (4.8) with (4.5), we have $\lim _{k \rightarrow+\infty} V_{n_{k}}=0$. Therefore, we have $\hat{V}=0$, which yields $\lim _{k \rightarrow+\infty} I_{n}=0$. Since $\lim _{k \rightarrow+\infty} N_{n}=\frac{A}{\mu}$, we have $\lim _{k \rightarrow+\infty} R_{n}=\frac{p A}{\mu}$.

Finally, we will prove that if $\mathcal{R}_{0} \leq 1$, then $E_{0}^{d}$ is uniformly stable. First, we consider the case that there exists a nonnegative integer $n_{1}$ such that $S_{n+1}>\bar{S}=\frac{(1-p) A}{\mu}$ for any $n \geq n_{1}$. From the first equation of (3.1), we have that for any $n>n_{1}$,

$$
\frac{S_{n+1}-S_{n}}{\phi}=(1-p) A-\mu S_{n+1}-\beta S_{n+1} I_{n} \leq 0,
$$

then, for any $n>n_{1}$, we obtain

$$
\frac{(1-p) A}{\mu} \leq S_{n+1} \leq S_{n} \leq S_{n_{1}}
$$

which implies that for any $n>n_{1}$,

$$
\left|S_{n+1}-\frac{(1-p) A}{\mu}\right| \leq\left|S_{n_{1}}-\frac{(1-p) A}{\mu}\right| .
$$

Meanwhile, from the second equation of model (3.1), we have that for any $n \geq n_{0}$,

$$
I_{n+1} \leq \frac{I_{n}\left(1+\beta \phi\left(\frac{(1-p) A}{\mu}+\epsilon\right)\right)}{1+(\mu+\gamma) \phi} .
$$

From the third equation of model (3.1), it is not difficult to obtain

$$
R_{n+1}-\frac{A p}{\mu}=\frac{\left(R_{n}-\frac{A p}{\mu}\right)}{1+\mu \phi}+\frac{\gamma \phi I_{n+1}}{1+\mu \phi}
$$

which implies that for any $n \geq n_{2}=\max \left\{n_{0}, n_{1}\right\}$ such that

$$
\left|R_{n+1}-\frac{A p}{\mu}\right| \leq\left|R_{n_{2}}-\frac{A p}{\mu}\right|\left(\frac{1}{1+\mu \phi}\right)^{n-n_{2}+1}+\frac{\gamma I_{n_{2}}}{\mu} .
$$

Next, we consider the case that there exists a nonnegative integer $n_{3}$ such that $S_{n_{3}} \leq \frac{(1-p) A}{\mu}$. Then, by the first equation of (3.1), we obtain

$$
S_{n_{3}+1} \leq \frac{S_{n_{2}}+A \phi(1-p)}{1+\mu \phi} \leq \frac{(1-p) A}{\mu} .
$$

Thus, we have, for $n \geq n_{3}, S_{n} \leq \bar{S}=\frac{(1-p) A}{\mu}$. Then, by the second equation of (3.1) and $\mathcal{R}_{0} \leq$ 1 , we have that for any $n \geq n_{3}$,

$$
I_{n+1}=\frac{I_{n}+\beta \phi S_{n+1} I_{n}}{1+(\mu+\gamma) \phi} \leq I_{n} \frac{1+(\mu+\gamma) \phi \mathcal{R}_{0}}{1+(\mu+\gamma) \phi} \leq I_{n} .
$$

Moreover, by the first equation of model (3.1), we have, for $n \geq n_{3}$,

$$
S_{n+1}-\frac{(1-p) A}{\mu}=\frac{S_{n}-\frac{(1-p) A}{\mu}}{1+\mu \phi}-\frac{\phi \beta S_{n+1} I_{n}}{1+\mu \phi},
$$


which implies that for $n \geq n_{3}$,

$$
\left|S_{n+1}-\frac{(1-p) A}{\mu}\right| \leq \frac{\left|S_{n_{3}}-\frac{(1-p) A}{\mu}\right|}{(1+\mu \phi)^{n-n_{3}+1}}+\frac{\beta(1-p) A}{\mu^{2}} I_{n_{3}} .
$$

By applying (4.11), we conclude that (4.10) holds for any $n \geq n_{3}$. Thus, from (4.9)-(4.12), we conclude that $E_{0}^{d}$ is uniformly stable. Hence, if $\mathcal{R}_{0} \leq 1, E_{0}^{d}$ is globally asymptotically stable.

\section{Global asymptotic stability of the endemic equilibrium $E_{d}^{*}$}

In this section, we mainly discuss the global dynamics of the endemic equilibrium $E_{d}^{*}$ of model (3.1). Before we prove the stability of the endemic equilibrium, we first give the following lemma.

Lemma $3[23,24]$ The quadratic equation $f(\lambda)=\lambda^{2}-B \lambda+C=0$ has two roots that satisfy $\left|\lambda_{i}\right|<1, i=1,2$, if and only if the following conditions are satisfied:

(i) $f(0)=C<1$,

(ii) $f(-1)=1+B+C>0$,

(iii) $f(1)=1-B+C>0$.

Theorem 6 If $\mathcal{R}_{0}>1$, then the endemic equilibrium point $E_{d}^{*}$ of discrete model (3.1) or equivalent (3.2) is locally asymptotically stable in $D_{h}$.

Proof Assuming that $\mathcal{R}_{0}>1$ and substituting the endemic equilibrium point $E_{d}^{*}$ to Jacobian matrix (4.1) lead to

$$
J\left(S_{d}^{*}, I_{d}^{*}\right)=\left(\begin{array}{cc}
\frac{1}{a} & -\frac{1}{a^{2}} \\
\frac{\mu \phi\left(\mathcal{R}_{0}-1\right)}{a b} & 1-\frac{\mu c \phi\left(\mathcal{R}_{0}-1\right)}{a^{2} b}
\end{array}\right),
$$

where $a=1+\phi\left(\mu+\beta I_{d}^{*}\right)>1, b=1+(\mu+\gamma) \phi>1, c=\beta \phi\left(S_{d}^{*}+(1-p) \phi A\right)>0$.

The characteristic equation of $J\left(S_{d}^{*}, I_{d}^{*}\right)$ is given by $f(\lambda)=\lambda^{2}-B \lambda+C=0$, straightforward calculation gives

$$
B=\frac{1}{a}+1-\frac{\mu c \phi\left(\mathcal{R}_{0}-1\right)}{a^{2} b}, \quad C=\frac{1}{a}=\frac{1}{1+\phi\left(\mu+\beta I_{d}^{*}\right)} .
$$

Obviously,

$$
0<C<1, \quad 1+B+C=\frac{4 a b+\mu \phi \mathcal{R}_{0}(2 a b-c)+\mu c \phi}{a^{2} b},
$$

where $2 a b-c=\left(1+\mu \phi \mathcal{R}_{0}\right)(2+(\mu+\gamma) \phi)>0$, that is, $1+B+C>0$. Finally, using the fact that $\mathcal{R}_{0}>1$, it is easy to obtain

$$
1-B+C=\frac{\mu c \phi\left(\mathcal{R}_{0}-1\right)}{a^{2} b}>0 .
$$

Hence all the conditions in Lemma 3 are satisfied when $\mathcal{R}_{0}>1$. This proves that when $\mathcal{R}_{0}>1$, then the endemic equilibrium point $E_{d}^{*}=\left(S_{d}^{*}, I_{d}^{*}, R_{d}^{*}\right)$ is locally asymptotically stable for any $h$. 
Next, we will prove the permanence of model (3.1) for $\mathcal{R}_{0}>1$. Similar to the result of McCluskey in $[25,26]$, we first give the following lemma.

Lemma 4 If $I_{n+1}<I_{n}$ holds, then $S_{n+1}<S_{d}^{*}$; inversely, if $S_{n+1} \geq S_{d}^{*}$ holds, then $I_{n+1} \geq I_{n}$, where

$$
S_{d}^{*}=\frac{\mu+\gamma}{\beta}, \quad I_{d}^{*}=\frac{\mu\left(\mathcal{R}_{0}-1\right)}{\beta} .
$$

Proof By the second equation of model (3.1), we obtain

$$
I_{n+1}-I_{n}=\phi\left(\beta S_{n+1} I_{n}-(\mu+\gamma) I_{n+1}\right) .
$$

If $S_{n+1} \geq S_{d}^{*}$ holds, then we have that

$$
I_{n+1}=\frac{1+\phi \beta S_{n+1}}{1+\phi(\mu+\gamma)} I_{n}<\frac{1+\phi \beta S_{d}^{*}}{1+\phi(\mu+\gamma)} I_{n}=I_{n}
$$

This completes the proof of Lemma 4.

Theorem 7 If $\mathcal{R}_{0}>1$, then for any solution of model (3.1) with the initial conditions that $S_{0}>0, I_{0}>0, R_{0}>0$

$$
\begin{aligned}
& \liminf _{n \rightarrow+\infty} S_{n} \geq \underline{S}:=\frac{\mu(1-p) A \phi}{\mu+\phi\left(\mu^{2}+\beta A\right)}, \\
& \liminf _{n \rightarrow+\infty} I_{n} \geq \underline{I}:=\left(\frac{1}{1+\phi(\mu+\gamma)}\right)^{l_{0}} I_{d}^{*},
\end{aligned}
$$

where the constant $l_{0}$ is sufficiently large such that $S_{d}^{*}<S^{\Delta}:=\frac{(1-p) A}{k^{*}}\left(1-\left(\frac{1}{1+\phi k^{*}}\right)^{l_{0}}\right)$.

Proof For any positive constant $\epsilon$, there exists a sufficiently large positive integer $n_{0}$ such that

$$
N_{n} \leq \frac{A}{\mu}+\epsilon \text { for } n \geq n_{0}
$$

By the first equation of models (3.1) and (5.3), we have that for any $n \geq n_{0}$,

$$
S_{n+1}=\frac{S_{n}+(1-p) \phi A}{1+\phi\left(\beta I_{n}+\mu\right)} \geq \frac{S_{n}+(1-p) \phi A}{1+\phi\left(\beta\left(\frac{A}{\mu}+\epsilon\right)+\mu\right)} .
$$

Since $\epsilon$ is arbitrary, we conclude that (5.1) holds. Now we prove that (5.2) holds. In fact, for any positive constant $q<1$, it is seen that

$$
S_{d}^{*}=\frac{\mu+\gamma}{\beta}=\frac{(1-p) A}{\mu+\beta I_{d}^{*}}<\frac{(1-p) A}{\mu+q \beta I_{d}^{*}} .
$$

We first claim that any solution of model (3.1) does not have the following property: there exists a nonnegative integer $n_{1}$ such that $I_{n} \leq q I_{d}^{*}$ for all $n \geq n_{1}$. Suppose on the contrary that there exists a solution $\left(S_{n}, I_{n}, R_{n}\right)$ of model (3.1) and a nonnegative $n_{1}$ such 
that $I_{n} \leq q I_{d}^{*}$ for $n \geq n_{1}$, it can be seen that for $n \geq n_{1}$,

$$
S_{n+1} \leq \frac{S_{n}+(1-p) \phi A}{1+\phi\left(\beta q I_{d}^{*}+\mu\right)}=\frac{S_{n}}{1+\phi k^{*}}+\frac{(1-p) \phi A}{1+\phi k^{*}} .
$$

Consequently, for $n \geq n_{1}$, we have

$$
\begin{aligned}
S_{n+1} & \geq\left(\frac{1}{1+\phi k^{*}}\right)^{n+1-n_{1}} S_{n_{1}}+\frac{(1-p) \phi A}{1+\phi k^{*}} \sum_{l=0}^{n-n_{1}}\left(\frac{1}{1+\phi k^{*}}\right)^{l} \\
& \geq \frac{(1-p) A}{k^{*}}\left(1-\left(\frac{1}{1+\phi k^{*}}\right)^{n-n_{1}+1}\right) .
\end{aligned}
$$

Since $\frac{(1-p) A}{k^{*}}>S_{d}^{*}$, therefore, there exists a positive $l_{0}$ such that for all $l \geq l_{0}$,

$$
\frac{(1-p) A}{k^{*}}\left(1-\left(\frac{1}{1+\phi k^{*}}\right)^{l}\right)>S_{d}^{*}
$$

We hence set

$$
S^{\Delta}=\frac{(1-p) A}{k^{*}}\left(1-\left(\frac{1}{1+\phi k^{*}}\right)^{l_{0}}\right) \text {. }
$$

Thus, by applying Lemma 1 , we have that $I_{n+1} \geq I_{n}$ for all $n \geq n_{1}+l_{0}+1$.

Furthermore, for the sequence $\left\{V_{n}\right\}_{0}^{+\infty}$ defined by (4.5), we have that for $n \geq n_{1}+l_{0}-1$,

$$
\begin{aligned}
V_{n+1}-V_{n} & =S_{n+2} I_{n+1} \beta \phi-(\mu+\gamma) I_{n+1} \phi \\
& \geq\left(\beta S^{\Delta}-(\mu+\gamma)\right) \phi I_{n_{1}+l_{0}-1} .
\end{aligned}
$$

Since $\beta S^{\Delta}-(\mu+\gamma) \phi>0$, this leads to $\lim _{n \rightarrow+\infty} V_{n}=\infty$, which yields a contradiction. Hence the claim is proved.

By the claim above, we are left to consider two possibilities. First, $I_{n} \geq q I^{*}$ for all $n$ sufficiently large. If this case holds, we get the conclusion of the proof. Second, we investigate the case that $I_{n}$ oscillates about $q I^{*}$ for all $n$ sufficiently large. Let $N_{1}<N_{2}$ be sufficiently large such that

$$
I_{N_{1}}, I_{N_{2}}>q I_{d}^{*}, \quad I_{n} \leq q I_{d}^{*} \quad \text { for } N_{1}<n<N_{2}
$$

By the second equation of model (3.1), we obtain, for $n>N_{1}$,

$$
I_{n+1} \geq\left(\frac{1}{1+\phi(\mu+\gamma)}\right)^{n-N_{1}+1} I_{N_{1}} \geq\left(\frac{1}{1+\phi(\mu+\gamma)}\right)^{n-N_{1}+1} q I_{d}^{*}
$$

Thus, we have that for all $N_{1} \leq n \leq N_{1}+l_{0}-1$,

$$
I_{n+1} \geq\left(\frac{1}{1+\phi(\mu+\gamma)}\right)^{l_{0}} q I_{d}^{*}=q \underline{I} .
$$

If $N_{2} \geq N_{1}+l_{0}$, then by applying a similar discussion above, we obtain $I_{n+1} \geq \underline{I}$ for all $N_{1}+$ $l_{0} \leq n \leq N_{2}-1$. We hence prove that $I_{n} \geq q \underline{I}$ for all $N_{1} \leq n \leq N_{2}$. Since the interval $\left[N_{1}, N_{2}\right]$ 
is arbitrarily chosen, we conclude that $I_{n} \geq q \underline{I}$ for all $n$ sufficiently large. Meanwhile, since $q$ is also arbitrary, we conclude that $\lim _{n \rightarrow+\infty} I_{n} \geq \underline{I}$. This completes the proof.

By Theorem 6, we easily obtain the permanence of model (3.1) for $\mathcal{R}_{0}>1$. Next, by constructing the Lyapunov function, we will prove that $E_{d}^{*}$ is globally asymptotically stable for $\mathcal{R}_{0}>1$. Consider the Lyapunov function as follows:

$$
W_{n}=\frac{1}{\beta I_{d}^{*}} g\left(\frac{S_{n}}{S_{d}^{*}}\right)+\frac{1+\beta S_{d}^{*}}{\beta S_{d}^{*}} g\left(\frac{I_{n}}{I_{d}^{*}}\right),
$$

where the function $g(x)=x-1-\ln x$. First, by calculating, we have

$$
\begin{aligned}
g\left(\frac{S_{n+1}}{S_{d}^{*}}\right)-g\left(\frac{S_{n}}{S_{d}^{*}}\right) & =\frac{S_{n+1}}{S_{d}^{*}}-\frac{S_{n}}{S_{d}^{*}}-\ln \frac{S_{n+1}}{S_{n}} \\
& \leq \frac{S_{n+1}-S_{n}}{S_{d}^{*}}-\frac{S_{n+1}-S_{n}}{S_{n+1}} \\
& =\frac{S_{n+1}-S_{d}^{*}}{S_{d}^{*} S_{n+1}}\left(S_{n+1}-S_{n}\right) \\
& =\frac{S_{n+1}-S_{d}^{*}}{S_{d}^{*} S_{n+1}} \phi\left((1-p) A-\beta S_{n+1} I_{n}-\mu S_{n+1}\right) \\
& =\frac{S_{n+1}-S_{d}^{*}}{S_{d}^{*} S_{n+1}} \phi\left(\beta S_{d}^{*} I_{d}^{*}+\mu S_{d}^{*}-\beta S_{n+1} I_{n}-\mu S_{n+1}\right) \\
& =-\frac{\mu\left(S_{n+1}-S_{d}^{*}\right)^{2}}{S_{d}^{*} S_{n+1}}+\phi \beta I_{d}^{*}\left(1-\frac{S_{d}^{*}}{S_{n+1}}\right)\left(1-\frac{S_{n+1}}{S_{d}^{*}} \frac{I_{n}}{I_{d}^{*}}\right) .
\end{aligned}
$$

In the same way, we have

$$
\begin{aligned}
g\left(\frac{I_{n+1}}{I_{d}^{*}}\right)-g\left(\frac{I_{n}}{I_{d}^{*}}\right) & =\frac{I_{n+1}}{I_{d}^{*}}-\frac{I_{n}}{I_{d}^{*}}-\ln \frac{I_{n+1}}{I_{n}} \\
& \leq \frac{I_{n+1}-I_{n}}{I_{d}^{*}}-\frac{I_{n+1}-I_{n}}{I_{n+1}} \\
& =\frac{I_{n+1}-I_{d}^{*}}{I_{d}^{*} I_{n+1}}\left(I_{n+1}-I_{n}\right) \\
& =\frac{I_{n+1}-I_{d}^{*}}{I_{d}^{*} I_{n+1}} \phi\left(\beta S_{n+1} I_{n}-(\gamma+\mu) I_{n+1}\right) \\
& =\frac{I_{n+1}-I_{d}^{*}}{I_{d}^{*} I_{n+1}} \phi\left(\beta S_{n+1} I_{n}-\beta S_{d}^{*} I_{n+1}\right) \\
& =\phi \beta S_{d}^{*}\left(1-\frac{I_{d}^{*}}{I_{n+1}}\right)\left(\frac{S_{n+1}}{S_{d}^{*}} \frac{I_{n}}{I_{d}^{*}}-\frac{I_{n+1}}{I_{d}^{*}}\right) .
\end{aligned}
$$

From (5.4) and (5.5), we have

$$
\begin{aligned}
\frac{W_{n+1}-W_{n}}{\phi}= & \left(1-\frac{S_{d}^{*}}{S_{n+1}}\right)\left(1-\frac{S_{n+1}}{S_{d}^{*}} \frac{I_{n}}{I_{d}^{*}}\right)-\frac{\mu\left(S_{n+1}-S_{d}^{*}\right)^{2}}{\beta I_{d}^{*} S_{d}^{*} S_{n+1}} \\
& +\left(1-\frac{I_{d}^{*}}{I_{n+1}}\right)\left(\frac{S_{n+1}}{S_{d}^{*}} \frac{I_{n}}{I_{d}^{*}}-\frac{I_{n+1}}{I_{d}^{*}}\right)
\end{aligned}
$$




$$
\begin{aligned}
& =-\frac{\mu\left(S_{n+1}-S_{d}^{*}\right)^{2}}{\beta I_{d}^{+} S_{d}^{*} S_{n+1}}-\left(-2+\frac{S_{d}^{*}}{S_{n+1}}+\frac{S_{n+1} I_{n}}{S_{d}^{*} I_{n+1}}+\ln \frac{I_{n+1}}{I_{d}^{*}}-\ln \frac{I_{n}}{I_{d}^{*}}\right) \\
& =-\frac{\mu\left(S_{n+1}-S_{d}^{*}\right)^{2}}{\beta I_{d}^{*} S_{d}^{*} S_{n+1}}-\left(g\left(\frac{S_{d}^{*}}{S_{n+1}}\right)+g\left(\frac{S_{n+1} I_{n}}{S_{d}^{*} I_{n+1}}\right)\right),
\end{aligned}
$$

which implies that $\left\{W_{n}\right\}_{0}^{+\infty}$ is a monotone decreasing sequence. We easily get

$$
\lim _{n \rightarrow+\infty} W_{n}=\hat{W} .
$$

Then $\lim _{n \rightarrow+\infty}\left(W_{n+1}-W_{n}\right)=0$, which combined with (5.6) implies that

$$
\lim _{n \rightarrow+\infty} S_{n}=S_{d}^{*}, \quad \lim _{n \rightarrow+\infty} \frac{I_{n}}{I_{n+1}}=1 .
$$

From the first equation of models (3.1) and (5.7), we have

$$
\lim _{n \rightarrow+\infty} I_{n}=\frac{\mu S_{d}^{*}+(1-p) A}{\beta S_{d}^{*}}=\frac{\mu\left(\mathcal{R}_{0}-1\right)}{\beta} .
$$

Notice that $W_{n} \leq W_{0}$, which implies that $E_{d}^{*}$ is uniformly stable. Finally, we hence obtain the theorem as follows.

Theorem 8 If $\mathcal{R}_{0}>1$, then the endemic equilibrium $E_{d}^{*}$ for model (3.1) or equivalent (3.2) is globally asymptotically stable.

\section{Numerical simulations}

In this section, numerical simulations will be given to verify theoretical results obtained in the previous section. The simulation is performed using MATLAB software.

(i) We choose $A=28, \mu=0.7, \gamma=15, p=0.5, \beta=0.7$ and $\phi=0.2$. By calculation, we have that $\mathcal{R}_{0}=0.8917$ and the endemic equilibrium $E_{d}^{0}=(16,0,24)$. According to Theorem 5, the disease-free equilibrium $E_{d}^{0}$ of discrete model (3.1) or equivalent (3.2) is globally stable, which is shown in Figure 1.

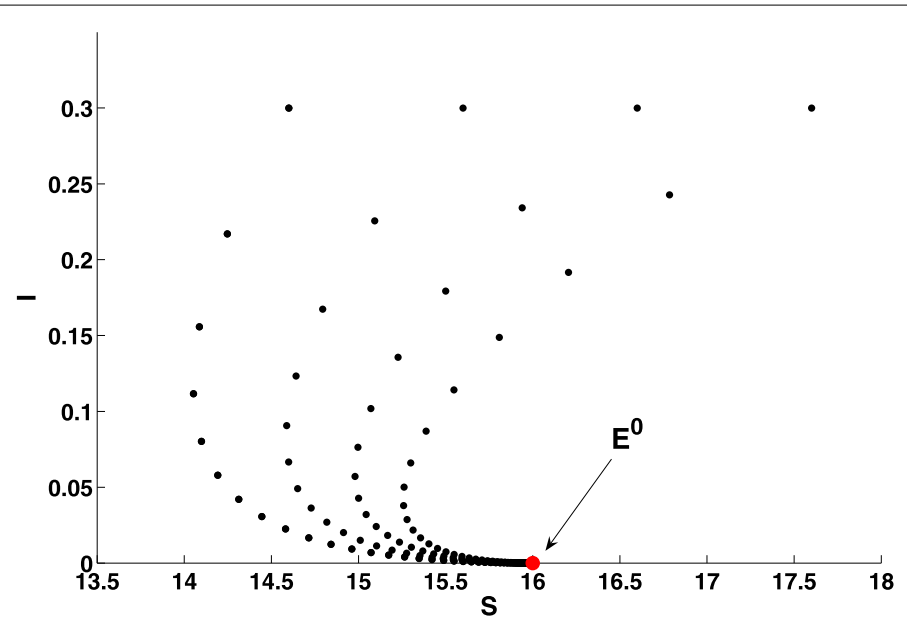

Figure 1 The solutions $(S(t), I(t), R(t))$ of model (3.1) or equivalent (3.2) are globally asymptotically stable and converge to the disease-free equilibrium $E_{d}^{0}$, when $\mathcal{R}_{0} \leq 1$. 


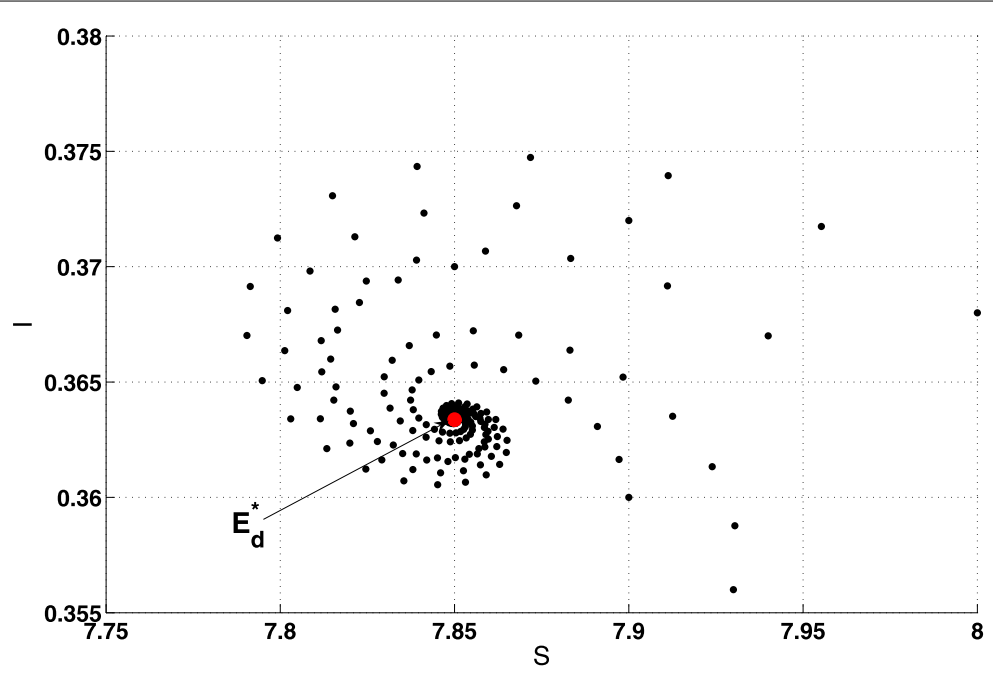

Figure 2 The solutions $(S(t), I(t), R(t))$ of model (3.1) or equivalent (3.2) are globally asymptotically stable and converge to the disease-free equilibrium $E_{d}^{*}$, when $\mathcal{R}_{0}>1$.

(ii) Assuming the following parameter values: $A=28, \mu=0.7, \gamma=15, p=0.5, \beta=2$ and $\phi=0.2$, by calculation, we have $\mathcal{R}_{0}=2.0382$ and the endemic equilibrium $E_{d}^{*}=(7.85,0.3634,24.5866)$. According to Theorem 8 , the endemic equilibrium $E_{d}^{*}=\left(S_{d}^{*}, I_{d}^{*}, R_{d}^{*}\right)$ of discrete model (3.1) or equivalent (3.2) is globally stable, which is depicted in Figure 2.

\section{Conclusion}

In this paper, we have proposed a discrete-time analogue of the continuous SIR epidemic model of childhood diseases with constant vaccination strategy which is derived by the NSFD scheme of Michens. In order to obtain the permanence of model (3.1) for $\mathcal{R}_{0}>1$, we offer Lemma 4. Applying the discrete Lyapunov functional technique (see $[25,26]$ ) for both cases $\mathcal{R}_{0} \leq 0$ and $\mathcal{R}_{0}>1$, it shown that the global dynamics of this discretetime analogue of the continuous SIR epidemic model is fully determined only by the basic reproduction number $\mathcal{R}_{0}$. This shows dynamical consistency between the discrete SIR epidemic model and its corresponding continuous model. The NSFD scheme constructed in this paper is for the SIR epidemic model with constant vaccination strategy. For our future work, we will consider an epidemic model with varying vaccination strategy.

Competing interests

The authors declare that they have no competing interests.

Authors' contributions

The authors declare that the study was realized in collaboration with the same responsibility. All authors read and approved the final manuscript.

\section{Author details}

${ }^{1}$ Department of Applied Mathematics, Nanjing University of Science and Technology, Nanjing, 210094, People's Republic of China. ${ }^{2}$ College of Sciences, Shihezi University, Shihezi, 832000, People's Republic of China. ${ }^{3}$ Xinjiang Institute of Engineering, Urumqi, 830091, People's Republic of China. ${ }^{4}$ Department of Medical Engineering and Technology, Xinjiang Medical University, Urumqi, 830011, People's Republic of China.

\section{Acknowledgements}

The work was supported by the National Natural Science Foundation of P.R. China $(11201399,11301451)$ and the Natural Science Foundation of Shihezi University (2013ZRKXYQ-YD05). 
Received: 10 December 2013 Accepted: 4 June 2014 Published: 24 June 2014

\section{References}

1. Arafa, AAM, Rida, SZ, Khalil, M: Solutions of fractional order model of childhood diseases with constant vaccination strategy. Math. Sci. Lett. 1, 17-23 (2013)

2. Cui, Q, Yang, X, Zhang, Q: An NSFD scheme for a class of SIR epidemic model with vaccination and treatment. J. Differ. Equ. Appl. 20, 416-422 (2014)

3. Li, J, Zhang, J, Ma, Z: Global analysis of some epidemic models with general contact rate and constant immigration. Appl. Math. Mech. 4, 396-404 (2004)

4. Makinde, OD: Adomian decomposition approach to a SIR epidemic model with constant vaccination strategy. Appl. Math. Comput. 184, 842-848 (2007)

5. Mickens, RE: A SIR-model with square-root dynamics: an NSFD scheme. J. Differ. Equ. Appl. 16, $209-216$ (2010)

6. Wang, L, Cui, Q, Teng, Z: Global dynamics in a class of discrete-time epidemic models with disease courses. Adv. Differ. Equ. 2013, 57 (2013). http://www.advancesindifferenceequations.com/content/2013/1/57

7. Yildirim, A, Cherruault, Y: Analytical approximate solution of a SIR epidemic model with constant vaccination strategy by homotopy perturbation method. Kybernetes 38, 1566-1575 (2009)

8. Hu, Z, Teng, Z, Jiang, H: Stability analysis in a class of discrete SIRS epidemic models. Nonlinear Anal., Real World Appl. 13, 2017-2033 (2012)

9. Suryanto, A: A dynamically consistent nonstandard numerical scheme for epidemic model with saturated incidence rate. Int. J. Math. Comput. 13, 112-123 (2011)

10. Suryanto, A: Stability and bifurcation of a discrete SIS epidemic model with delay. In: Proceedings of the 2nd International Conference on Basic Sciences, Indonesia, pp. 1-6 (2012)

11. Mickens, RE: Nonstandard Finite Difference Model of Differential Equations. World Scientific, Singapore (1994)

12. Mickens, RE: Application of Nonstandard Finite Difference Schemes. World Scientific, Singapore (2000)

13. Mickens, RE: Dynamic consistency: a fundamental principle for constructing nonstandard finite difference schemes for differential equations. J. Differ. Equ. Appl. 11, 645-653 (2005)

14. Mickens, RE: Calculation of denominator functions for nonstandard finite difference schemes for differentia equations satisfying a positivity condition. Numer. Methods Partial Differ. Equ. 3, 528-534 (2012)

15. Mickens, RE: Nonstandard finite difference schemes for differential equations. J. Differ. Equ. Appl. 8, $823-847$ (2002)

16. Mickens, RE: Numerical integration of population models satisfying conservation laws: NSFD methods. J. Biol. Dyn. 1, 427-436 (2007)

17. Ding, X: A non-standard finite difference scheme for an epidemic model with vaccination. J. Differ. Equ. Appl. 19, 179-190 (2013)

18. Mickens, RE, Washington, T: A note on an NSFD scheme for a mathematical model of respiratory virus transmission. J. Differ. Equ. Appl. 8, 525-529 (2012)

19. Suryanto, A, Kusumawinahyu, WM, Darti, I, Yanti, I: Dynamically consistent discrete epidemic model with modified saturated incidence rate. Comput. Appl. Math. 32, 373-383 (2013)

20. Ross, SL: Differential Equations. Blaisdell, Waltham (1964)

21. Strogatz, SH: Nonlinear Dynamics and Chaos. Addison-Wesley, Reading (1994)

22. Izzo, G, Muroya, Y, Vecchio, A: A general discrete time model of population dynamics in the presence of an infection. Discrete Dyn. Nat. Soc. 2009, 143019 (2009). doi:10.1155/2009/143019

23. Brauer, F, Castillo-Chavez, C: Mathematical Models in Population Biology and Epidemiology. Springer, New York (2001)

24. Elaydi, S: An Introduction to Difference Equations, 3rd edn. Springer, New York (1992)

25. McCluskey, CC: Global stability for an SEIR epidemiological model with varying infectivity and infinite delay. Math. Biosci. Eng. 6, 603-610 (2009)

26. McCluskey, CC: Complete global stability for an SIR epidemic model with delay - distributed or discrete. Nonlinear Anal., Real World Appl. 10, 55-59 (2010)

doi:10.1186/1687-1847-2014-172

Cite this article as: Cui et al.: An NSFD scheme for SIR epidemic models of childhood diseases with constant vaccination strategy. Advances in Difference Equations 2014 2014:172

\section{Submit your manuscript to a SpringerOpen ${ }^{\ominus}$ journal and benefit from:}

- Convenient online submission

- Rigorous peer review

- Immediate publication on acceptance

Open access: articles freely available online

- High visibility within the field

- Retaining the copyright to your article 